\title{
Interrelación entre riesgo e innovación: percepción del riesgo por gestores de proyectos
}

\author{
Rosalba Martínez Hernández ${ }^{1 *}$, María del Pilar Pastor Pérez ${ }^{1}$
}

\begin{abstract}
Title: Relationship between risk and innovation: Risk perception by project managers
In an environment of uncertainty in the organizations, risk aversion from decision makers can be a rational behaviour but reduces the ability to obtain innovate and then risk of failure increases. This paper analyses under an exploratory approach, the perception of the risk identified by Mexican innovation project leaders, in multinational companies. The results obtained from thirteen cases indicate that, there is no risk aversion and that the association between a higher level of profitability and greater risk is recognized, but that simple or intuitive methods are often used to manage risk. This highlights the need to train managers and executives with a wide risk and risk management overview, innovation and its relationship.
\end{abstract}

Keywords: Risk; Innovation; Risk Taking; Project Management; Management of Technological Innovation and R\&D.

Resumen: En un entorno de incertidumbre, la aversión al riesgo por parte de los tomadores de decisiones en las organizaciones puede ser un comportamiento racional, pero reduce la capacidad de innovar y se incrementa la probabilidad de fracaso. Este trabajo de tipo exploratorio analiza la percepción del riesgo identificada por líderes de proyectos de innovación, en empresas multinacionales. Los resultados obtenidos a partir de trece casos en México, indican que no existe aversión al riesgo y se reconoce la asociación entre un mayor nivel de rentabilidad y mayor riesgo, pero con frecuencia se recurre a métodos simples para gestionar los riesgos en los proyectos. Esto enfatiza la necesidad de formar gestores con una visión amplia del riesgo, su gestión y relación con la innovación.

Palabras clave: Riesgo; Innovación; Toma de Riesgo; Gestión de Proyectos; Gestión de Innovación Tecnológica y de la I+D.

Submitted: March 2 $2^{\text {nd }}, 2017$ / Approved: April $6^{\text {th }}, 2018$

\section{Introduction}

La evolución de un entorno complejo ha configurado cada vez más, un escenario de riesgos para todo tipo de organizaciones. En el caso de las empresas, la innovación es fuente fundamental de ventajas competitivas sustentables (Porter, 1990), lo que reduce el riesgo de que se vean desplazadas por otras firmas y por tanto de quedar fuera del mercado. Sin embargo, a pesar del consenso en la relación innovación-competitividad, innovar no es la panacea, porque es a su vez una fuente de riesgos para la organización.

Bajo este panorama de incertidumbre, una mayor aversión al riesgo por parte de los tomadores de decisiones en las organizaciones puede ser un comportamiento racional. No obstante, se reduce la capacidad de obtener resultados innovadores y por tanto incrementa el riesgo de fracaso organizacional. Ante la necesidad estratégica de invertir en innovación y adoptar prácticas que ayuden a facilitar procesos innovadores, es necesario contribuir a desarrollar modelos y procedimientos para gestionar riesgos en actividades innovadoras (Verbano \& Venturini, 2013).

El análisis de la actitud de los líderes de proyectos de innovación es relevante y no se ha estudiado con suficiencia. Los determinantes de dicha actitud son diversos y tienen su origen, tanto en el contexto en el que se desenvuelve el individuo, como en las características personales (March \& Shapira, 1987; Peterson, Smith, Martorana, \& Owens, 2003; García-Granero, Llopis, Fernández-Mesa, \& Alegre, 2015). Sin duda, la expectativa de una rentabilidad elevada favorece que los tomadores de decisiones con reducida aversión al riesgo, valoren más los beneficios que las pérdidas potenciales de la innovación. Sin embargo, entre dos alternativas con el mismo valor esperado, los agentes económicos adversos al riesgo, eligen una alternativa con un resultado menos incierto (Hartono et al., 2014). En cuanto a las consecuencias, la relación entre la propensión a asumir riesgos y la innovación es positiva; es decir, la preferencia de los gestores por comportamientos arriesgados está asociada positivamente con el logro de elevados resultados innovadores (Ling, Simsek, Lubatkin, \& Veiga, 2008; Wu, 2008; García-Granero et al., 2015).

Se cuenta con evidencia en el tema riesgos y proyectos en países como Nueva Zelanda, Israel, Japón e Indonesia, e incluso en países latinoamericanos como es el caso de Brasil (Zwikael \& Ahn, 2011; Rabechini \& Monteiro, 2013; Hartono, et al., 2014); sin embargo,los trabajos sobre la percepción del riesgo y la actividad innovadora de las empresas, son escasos en las economías emergentes, donde el estudio de los sistemas de innovación y el comportamiento innovador de sus actores, se ha centrado más bien en medir las capacidades y resultados. Por tanto, encontramos un interesante campo de análisis en la exploración del papel que juega la percepción del riesgo de los tomadores de decisiones y la innovación en las empresas.

Facultad de Contaduría y Administración, Universidad Autónoma de San Luis Potosí, San Luis Potosí, México.

*Autor de correspondencia: rosalba.martinez@uaslp.mx 
En este contexto, se llevó a cabo una investigación con el objetivo explorar la percepción del riesgo que tienen líderes de proyectos de innovación. Aunque el levantamiento de información se realizó en México, dada la escasez de trabajos de este tipo, los resultados pueden tomarse como referente para otras evaluaciones en América Latina. Los resultados ponen de relieve la necesidad de formar gestores y directivos con una visión amplia del riesgo, la innovación y su relación. Para ello, a continuación, el documento se estructura en cuatro apartados. En primer lugar, como marco de referencia, se analizan los significados e implicaciones de los conceptos de riesgo e innovación, a fin de entender su naturaleza y abordar su interrelación. Después, se resumen los aspectos correspondientes a la metodología. Posteriormente, se presentan los resultados, y, como parte final se exponen las conclusiones.

\section{Riesgo}

De acuerdo al Project Management Institute (PMI), el riesgo es un evento o condición incierta que, de producirse, tiene un efecto positivo o negativo en uno o más de los objetivos del proyecto, tales como el alcance, el cronograma, el costo y la calidad (PMIPMBOK, 2013). Por su parte, la norma ISO 31000 (2009) define el riesgo como la exposición a las consecuencias de incertidumbre o desviaciones potenciales de lo que había sido previsto. En el contexto de un proyecto, el riesgo tiene su origen en el campo de las incertidumbres, mismas que se presentan en mayor o menor medida en todos los proyectos. A este respecto, Rabechini y Monteiro (2013) distinguen el concepto de incertidumbre, del concepto de riesgo, por la posibilidad de establecer la probabilidad de un evento, especificando que, la incertidumbre no permite asociar valores de probabilidad numérica y se carece de conocimiento respecto de las consecuencias de un evento.

En este sentido, se distinguen dos formas de abordar el riesgo (Holton, 2004). La primera sigue las interpretaciones objetivas que se han desarrollado con base en la medición del riesgo. La segunda, a partir de las interpretaciones subjetivas de la probabilidad, que surgen de interpretaciones y creencias humanas caracterizadas por los individuos y que, por tanto, no pueden ser medidas de manera cuantitativa. Adicionalmente, es posible identificar diferentes perspectivas del riesgo. La perspectiva unidimensional, que relaciona el riesgo con una pérdida (Holton, 2004) y, la perspectiva dual o neutral del riesgo, que da cabida a considerar una expectativa en relación con un beneficio, y con ello el aspecto positivo del riesgo (Hillson, 2002; Hartono et al., 2014).

Con base en las diferentes perspectivas del riesgo, y el tipo de riesgos que presenta cada proyecto - técnico, operativo, organizacional, contractual, financiero, económico, político, entre otros-, se han identificado diferentes nociones acerca de la gestión de riesgos. Tradicionalmente, la gestión de riesgos se define como la función que desempeña la dirección, con el objetivo de proteger a la empresa contra las consecuencias adversas del riesgo, orientada así a la reducción de la severidad y variabilidad de las pérdidas, el lado negativo del riesgo (PwC \& IFAC, 1999). En el caso de la ISO 31000 se hace referencia a la gestión de riesgos como una disciplina clave para la toma de decisiones efectivas y la comunicación de los resultados dentro de las empresas. Su propósito es identificar los posibles problemas administrativos y técnicos antes de que ocurran, de manera que se puedan tomar medidas que reduzcan o eliminen la probabilidad y/o el impacto de estos problemas cuando se produzcan (ISO, 2009).

En el marco de la gestión de proyectos, la gestión de riesgos es un proceso integrado en el ciclo de vida del proyecto. Implica la definición de objetivos, la identificación de fuentes de incertidumbre, el análisis de estas incertidumbres y la formulación de respuestas gerenciales para desarrollar un equilibrio aceptable entre riesgos y oportunidades (Thevendran \& Mawdesley, 2004). El Project Management Institute (PMI, 2013) presenta la gestión de riesgos como una serie de procesos interconectados que involucran técnicas y herramientas específicas, con base en seis etapas: 1) planeación, 2) identificación, 3) análisis cualitativo, 4) análisis cuantitativo, 5) respuesta al riesgo, 6) supervisión y control.

Al percibir, entender y gestionar el riesgo, con base en un enfoque amplio, no sólo de peligro sino también de oportunidad, los gestores y directivos, tienen la posibilidad de explotar la incertidumbre a favor de la empresa. Una percepción amplia u holística constituye una herramienta útil para mejorar la capacidad de generar valor (ver figura I), porque permite a gestores y directivos una visión orientada hacia la progresión, que va de una gestión asociada con el cumplimiento y prevención, hasta desplazarse hacia una gestión que aprovecha las oportunidades derivadas de los riesgos y la conexión existente entre la gestión del riesgo y el valor de la empresa. 
Figura 1. Perspectiva de la Gestión de Riesgos: Integración del Crecimiento, Riesgo y Rendimiento

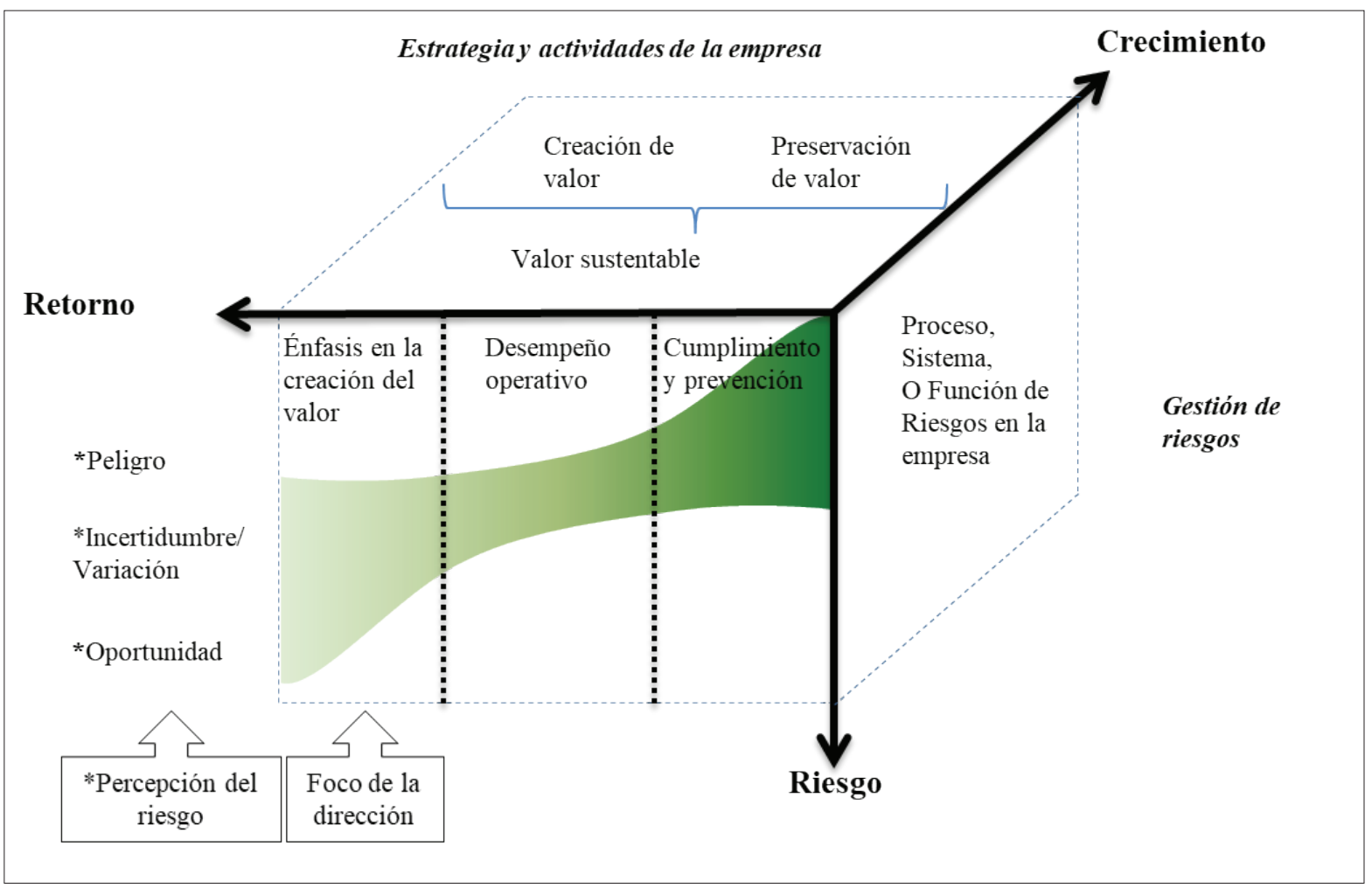

Fuente: Adaptado de PwC e IFAC (1999).

\section{Interrelación entre riesgo e innovación}

La innovación es un producto, proceso o método que se introduce al mercado y que posee algún grado de novedad, a partir del cual se distingue entre innovaciones radicales, que son aquellas que tienen un impacto significativo en un mercado, y otras que son de tipo incremental, (Organisation for Economic Co-operation and Development [OECD] \& Eurostat, 2005). En particular, la innovación tecnológica es, según la literatura sobre los modelos de crecimiento endógeno, esencial en el crecimiento económico a largo plazo (Benhabib, Perla, $\&$ Tonetti, 2014). Por esto, las diferencias en el crecimiento de los países pueden encontrarse en las debilidades y fortalezas de sus capacidades de innovación entre los que destacan el gasto en investigación y desarrollo tecnológico (I+D) y el capital humano.

Pasando a la perspectiva microeconómica, es importante observar los procesos que las empresas emprenden para innovar porque la innovación suele ser el resultado de proyectos que, más allá del tipo de gestión, tienen frecuentemente una alta tasa de fracaso. A este respecto, Bowers y Khorakian (2014) señalan que un gran número de proyectos de innovación se inician en la etapa de creatividad, y de estos, la mayoría se abandona en diversos momentos del proceso, con muy pocos proyectos sobrevivientes a través de la implementación, acentuando que siempre hay un grado significativo de incertidumbre.

En este contexto, la actitud de los gerentes de las organizaciones frente al riesgo es un asunto que no se debe menospreciar porque, más allá de los factores del entorno, puede ir en detrimento de la actividad innovadora de las organizaciones. Entre las motivaciones que impulsan a gestores y directivos empresariales a desarrollar proyectos de innovación, se identifican: el incremento de la cuota de mercado, la reducción de costos, y la mejora de la calidad de la oferta. Entre los factores que dificultan el proceso de innovación, se reconoce los costos de las actividades de $\mathrm{I}+\mathrm{D}$ y el riesgo inherente a estas, y destacan la escasez de financiamiento adecuado para este tipo de actividades (OECD \& Eurostat, 2005).Los proyectos innovadores, son fundamentales para que las empresas obtengan ventajas competitivas y tengan éxito en el mercado, sin embargo necesariamente implican decisiones y actividades arriesgadas. De ahí que la gestión de riesgos podría mejorar la capacidad de gestionar con éxito todas las etapas de los proyectos incluidas aquellas que son innovadoras (Verbano \& Venturini, 2013).

La probabilidad de éxito de un proyecto de innovación tecnológica es mayor, cuanto menor es el grado de innovación que se busca. Es decir, la innovación radical tiene una probabilidad de éxito menor, pero un potencial competitivo mayor que las innovaciones incrementales (Roussel, Saad, \& Erickson, 1991). Así, se tiene que los elevados niveles de riesgo asociados a los proyectos innovadores justifican la necesidad de implementar estrategias de gestión de riesgos (Vargas-Hernández, 2011). No obstante, una gestión inapropiada o exagerada puede cohartar la creatividad y mermar el desempeño innovador (Taplin \& Schymyck, 2005). No se trata de evitar el riesgo, sino de gestionarlo con el objetivo de minimizar el impacto en caso de que el proyecto innovador no tenga los resultados esperados (Bowers \& Khorakian, 2014). 
En general, la literatura sobre el tema acepta el papel crítico de la asunción de riesgos como motor de la innovación y de la orientación emprendedora de las organizaciones (Rauch, Wiklund, Lumpkin, \&
Frese, 2009; García-Granero et al., 2015). En este sentido, se tienen diferentes premisas que sustentan el binomio de riesgo e innovación (Ver Tabla 1).

Tabla 1. Premisas que Vinculan la Innovación y el Riesgo

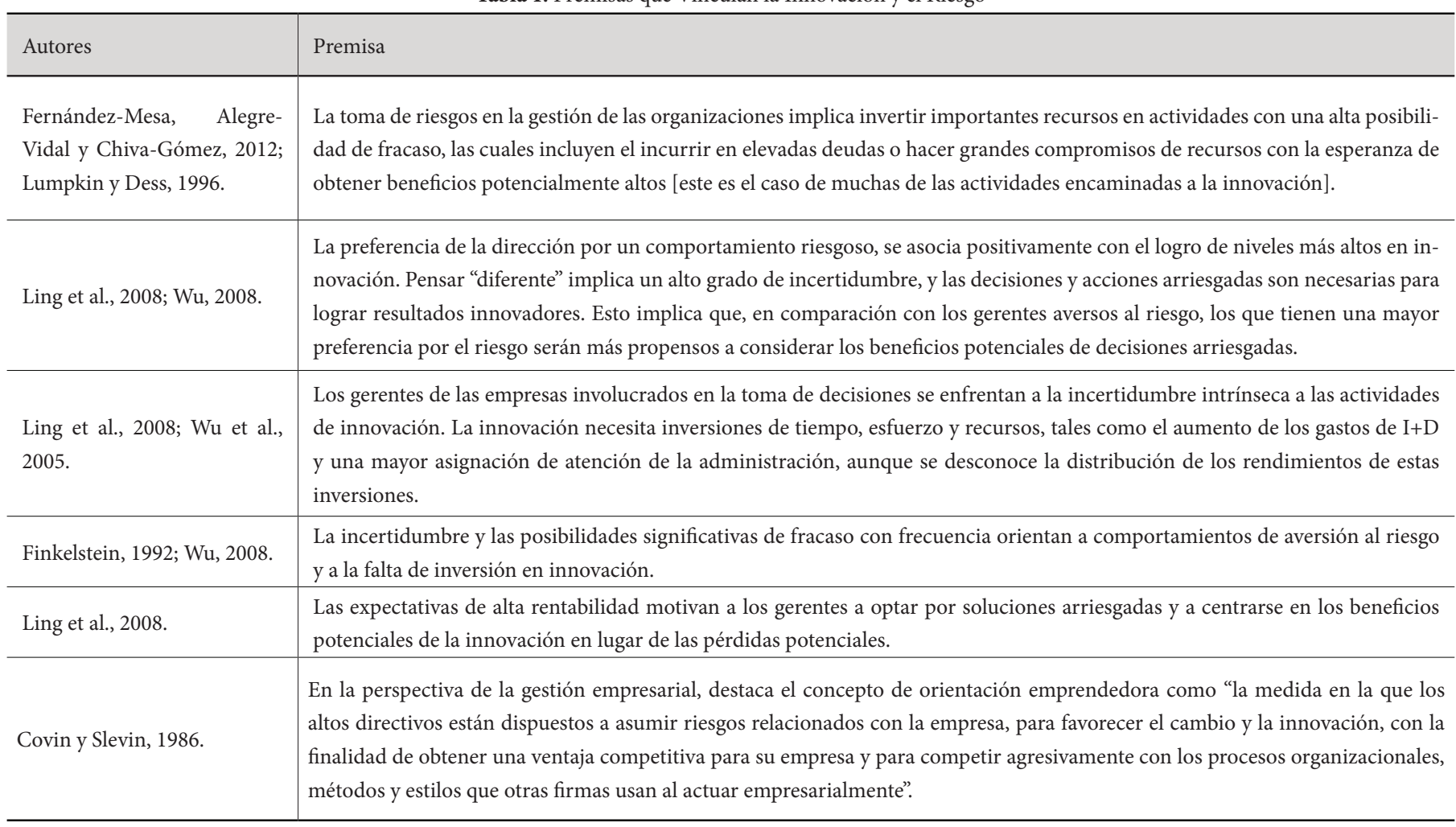

Fuente: elaboración propia con base en García-Granero et al. (2015).

Entre las conclusiones de lo que se publica sobre riesgo suelen derivarse recomendaciones prácticas para la gerencia; por ejemplo, Fernández-Mesa et al. (2012) afirman que los directivos deben mejorar la capacidad de aprendizaje organizacional, incluida la asunción de riesgos, porque esto tiene impactos positivos en el proceso de aprendizaje e innovación. A pesar de esto, se observa una carencia de estudios sobre percepción y gestión del riesgo en proyectos innovadores, a nivel de la práctica profesional A este respecto, se han realizado algunos en empresas de Estados Unidos, Suecia e Indonesia, con base en la perspectiva de gestores y directivos. Los resultados ponen de relieve un predominio de la perspectiva negativa del riesgo (March \& Shapira, 1987; MacCrimmon \& Wehrung, 1990; Riabacke, 2006; Hartono et al., 2014).

En lo que se refiere a la gestión de proyectos, se hace evidente el interés de la investigación de la gestión de riesgos a partir de la década de 1990 (Rabechini \& Monteiro, 2013). No obstante, se han publicado pocos artículos por año, denotando una tendencia creciente a partir del año 2006. La mayoría de los artículos, proceden de China y Reino Unido. El sector más analizado es la manufactura, mientras que una minoría de trabajos evalúa las empresas que fueron operadas por proyecto y de éstos solo unos cuantos consideran el tema de los riesgos en proyectos (Verbano \& Venturini, 2013).
Entre los análisis empíricos sobre la gestión de riesgos de proyectos, cabe señalar los realizados en Nueva Zelanda, Israel, Japón (Zwikael \& Ahn, 2011) y Brasil (Rabechini \& Monteiro, 2013). Los resultados revelan que incluso los niveles moderados de planificación de la gestión de riesgos, son suficientes para reducir el efecto negativo que tienen los niveles de riesgo. Asimismo, que la presencia de un responsable de la gestión de riesgos contribuye a la percepción de éxito del proyecto.

Finalmente, ante la escasa evidencia sobre el binomio risgo e innovación en los proyectos, Verbano y Venturini (2013) hacen un llamado para realizar trabajos en la línea de investigación de la gestión de risgos en proyectos innovadores. Con base en lo anterior, se enuncian cuatro hipótesis de tipo descriptivo:

H1: La percepción del riesgo para más del 50\% de los profesionales que lideran proyectos de innovación comprende solo una dimensión del riesgo, la negativa.

H2: La actitud ante el riesgo para más del 50\% de los líderes de los proyectos de innovación es moderada.

H3: Los líderes de los proyectos de innovación perciben que hay una relación entre el nivel de riesgo y el nivel de rentabilidad. 
H4: Más del 50\% de los líderes de proyectos de innovación, utilizan técnicas cuantitativas para el análisis de riesgos.

\section{Metodología}

El trabajo llevado a cabo es de carácter exploratorio y descriptivo, debido fundamentalmente a que el tamaño de la muestra es muy reducido. Este método es útil para generar conocimiento sobre temas poco desarrollados. Se trata de un estudio de corte transversal en el que se aplicó un cuestionario, entendido este como un instrumento que facilita la recolección de datos para describir prácticas y probar hipótesis, utilizado en la investigación experimental y/o para propósitos descriptivos. Para el diseño del mismo, nos basamos en el propuesto por Hartono et al. (2014), adaptado con base en la literatura sobre el tema y para el contexto mexicano (ver tabla 2).

Tabla 2. Cuestionario

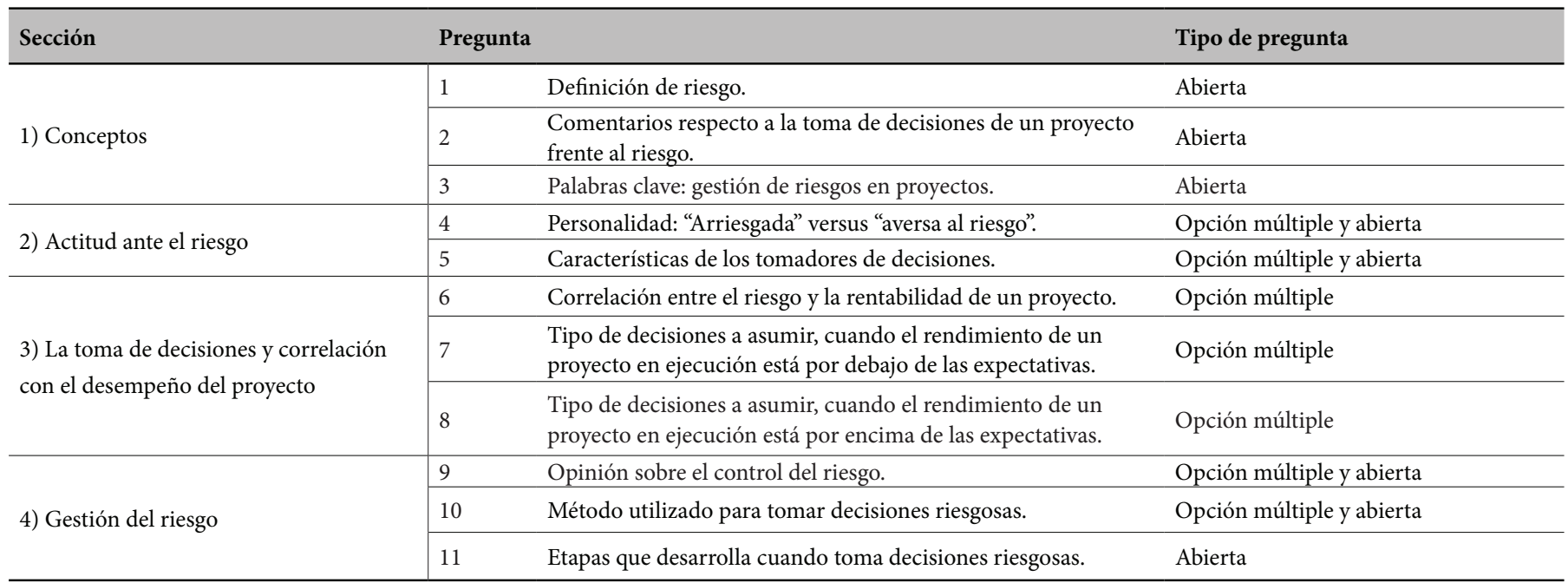

Fuente: elaboración propia con base en Hartono et al. (2014).

La población objeto de estudio son los tomadores de decisiones de proyectos de innovación. La muestra, seleccionada por conveniencia, está conformada por trece casos. Los informantes clave, fueron líderes de proyectos de doce empresas manufactureras y un centro de investigación, ubicados en San Luis Potosí, México. En el momento de la aplicación del cuestionario, enero de 2016, los informantes (ver tabla 3) participaban en sus respectivas organizaciones en la ejecución de algún proyecto con diferentes grados de innovación. El instrumento fue autoadministrado ante la presencia de los investigadores y para analizar las respuestas se recurrió a técnicas de análisis del discurso y estadística descriptiva.

Tabla 3. Líderes de Proyectos Innovadores Entrevistados

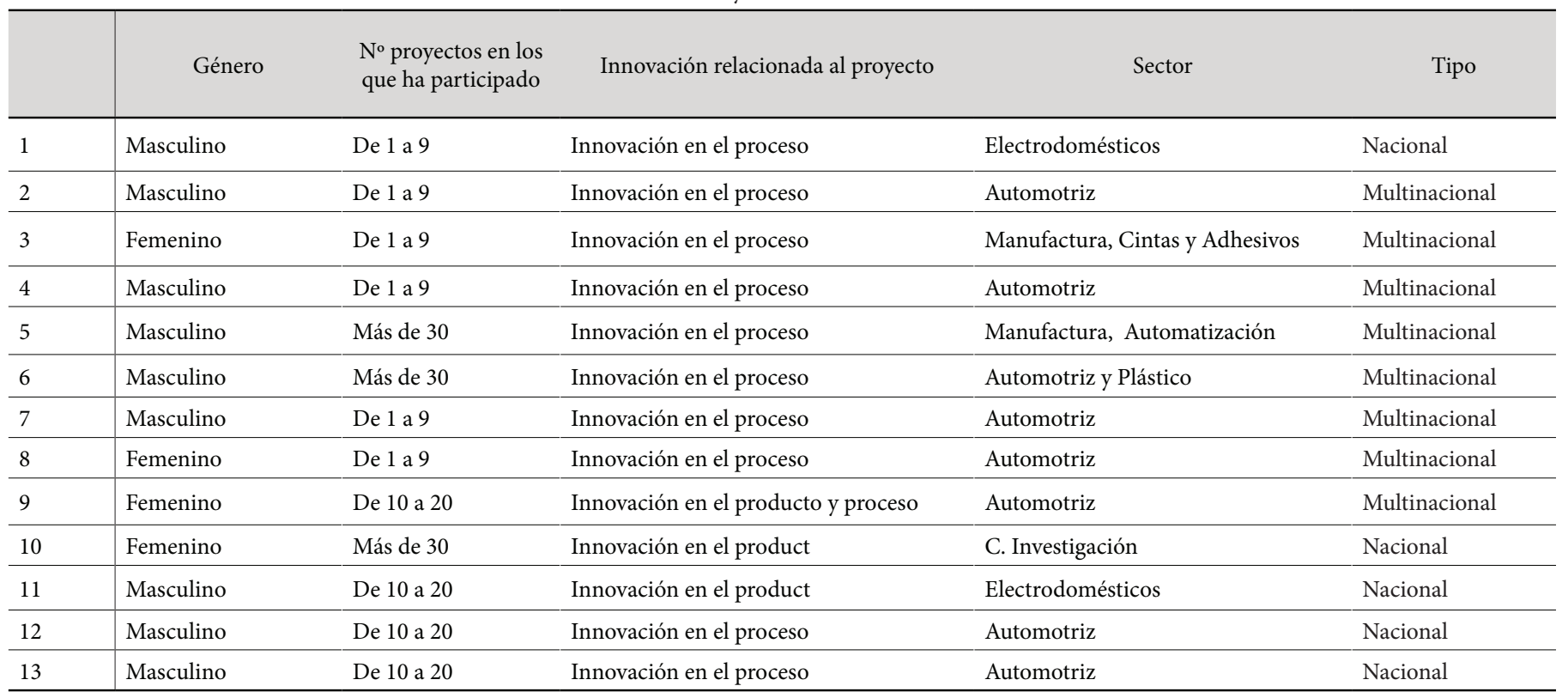

Fuente: elaboración propia con datos de los participantes entrevistados. 


\section{Resultados}

Definicion del Riesgo

Los resultados respecto a la definición del riesgo, indican que alrededor de la tercera parte de los participantes percibe el riesgo desde una vertiente unidimensional y orientada al lado negativo de este (38\%). El tono negativo está representado por expresiones como "evento que afecta de manera negativa", "evitar cumplir el objetivo", "impactan desfavorablemente a un objetivo deseado", "probabilidad de fracaso", "no tenga éxito".

Dichos resultados, están en linea con March y Shapira (1986) quienes indican que en Estados Unidos e Israel la mayoría de los gestores y directivos en la práctica de aquellos años, consideraban que el riesgo tenía como consecuencia resultados negativos. Lo que implica que gestores y directivos eran propensos a pasar por alto los resultados positivos como consecuencia de los riesgos. Además, Hartono et al. (2014), en Indonesia, identificaron un predominio del ángulo negativo del riesgo, representado por palabras clave como "pérdida" o "peor resultado".

Respecto al número de gestores que presentan una perspectiva dual o neutral del riesgo, es similar al de los gestores de proyectos que visualizan el riesgo como negativo (38\%). El tono neutral se identifica en las palabras "probabilidad de salirse de los planes", "cualquier cosa que afecte los requerimientos técnicos". Cabe señalar que el resto (23\%) de los participantes, definen el riesgo, mediante palabras que no muestran una relación semántica con las definiciones presentadas en la literatura. Esto se identifica mediante palabras como "son acciones", "actividad o servicio", "es el nivel de seguridad".

Estos resultados ponen de relieve la importancia de contribuir al entendimiento conceptual y las incertidumbres pues tal y como afirman Rabechini y Monteiro (2013), a partir del caso de Brasil, un mayor entendimiento de la gestión de riesgos en los proyectos, conlleva una mayor posibilidad de éxito en un proyecto.

Los resultados de las 63 palabras clave externadas por los participantes, indican referencia en primer lugar a los riesgos en los: 1) recursos económicos como son el "costo", e "impacto económico", 2) el "tiempo" y el 3) "éxito o fracaso" sobresaliendo en mayor medida el tono negativo, reflejado en palabras clave relacionadas con problemas y daños. A este respecto es evidente que en estos resultados se distinguen los elementos señalados en la definiciòn del riesgo propuestos por el PMI (PMBOK, 2013). Adicionalmente confirman los resultados de Zwikael y Ahn (2011) de que el tipo de industria, contexto del proyecto y el país, donde se ejecuta el proyecto, tienen un impacto significativo en los niveles percibidos del riesgo del proyecto y la intensidad de los procesos de gestión del riesgo.

Toma de decisiones frente a los riesgos

En relación con la toma de decisiones frente a los riesgos de un proyecto, se identifican tres diferentes grupos de comentarios. Los primeros, hacen referencia al tipo de proyecto y objetivos: "Generalmente dependiendo del tipo de proyecto y sus objetivos se tienen diferentes impactos al tomar decisiones en el proyecto que generan un riesgo de tipo económico, financiero, político o social, inclusive ambiental". "Dependiendo del tipo de proyecto, las decisiones y las prioridades pueden ser tomadas con base en aspectos financieros, de calidad, de presencia en el mercado, etc". "El alcance del proyecto y objetivo, determinan las prioridades".

El segundo grupo de comentarios está relacionado con que "en todos los proyectos existen riesgos con un impacto económico" asociados al "presupuesto considerado para el proyecto".

El tercer grupo de comentarios se asocia a la gestión del riesgo, centrados en las fases de evaluación y respuesta al riesgo: "La toma de decisiones para minimizar los impactos que pongan en riesgo el éxito del proyecto es crucial". "La toma de decisiones debe estar soportada en datos [...] para mitigar los errores y prevenir fallas". "Una decisión que se puede tomar para minimizar los riesgos es el subcontratar a especialistas que desarrollen una parte del proyecto específica. Otra manera de minimizar o mitigar los riesgos es "buscar asesoría especializada en los aspectos que no se conocen o dominan".

\section{Actitud ante el Riesgo}

En relación con la actitud ante el riesgo (ver tabla 4, panel A), la mayoría de los participantes se han auto percibido con una actitud moderada o neutra ante el riesgo, realizando afirmaciones tales como: "Generalmente analizo antes de actuar y no tomo riesgos innecesarios". "Soy una persona moderada, me gusta asumir en la mayoría de los casos, riesgos, sin embargo, de acuerdo a mi experiencia he aprendido a analizarlos y en ocasiones a rechazar situaciones o proyectos con base a mis análisis, datos..." "Analizo y pienso lógicamente antes de tomar una decisión, necesito basarme en datos...”. Respecto a las características del tomador de riesgos, se identifica que la que más sobresale en una persona que toma riesgos es el atrevimiento a cometer errores (38\%), tal y como se observa en la tabla 4, panel B.

En el mismo sentido, Riabacke (2006) evidencia que, en Suecia, el $50 \%$ de los encuestados son tomadores de riesgos, mientras que el $16.67 \%$ son reacios o aversos al riesgo. En constraste con los resultados que obtuvo Hartono et al. (2014), en los que se muestra que la mayoría de los encuestados han afirmado tener un tipo de actitud de riesgo moderada o situacional. Cabe señalar que también se aprecia que el nivel de riesgo percibido varía entre industrias y países. Reducir el nivel de riesgo es extremadamente importante en los proyectos (Zwikael \& Ahn 2011).

\section{Relación entre Riesgo y Desempeño}

Con base en los estudios previos, se esperarían respuestas que reflejen correlación positiva entre riesgo y rendimiento, es decir, "a mayor riesgo, mayor retorno o rendimiento". En efecto, la mayoría de los participantes, afirman que existe una relación positiva entre el nivel del riesgo y el rendimiento (ver tabla 5). De ahí que la mayoría de ellos generan expectativas de una mayor rentabilidad (o rendimiento) para proyectos de mayor riesgo. Estos resultados son consistentes con los obtenidos por Riabacke (2006), que indican que los directivos esperarían una mayor rentabilidad para las decisiones de mayor riesgo. 
Tabla 4. Actitud ante el Riesgo

\begin{tabular}{lcl}
\hline A) Autopercepción: actitud hacia el riesgo & \\
& $\mathbf{n}$ & $\%$ \\
Averso & 0 & $0 \%$ \\
Tomador de riesgos & 5 & $38 \%$ \\
Moderado o neutro & 8 & $62 \%$ \\
Otro & 0 & $0 \%$ \\
\hline Total & 13 & $100 \%$ \\
\hline
\end{tabular}

\begin{tabular}{lll}
\hline B) Características del tomador de riesgos & & \\
& n & $\%$ \\
Personalidades fuertes & 4 & $31 \%$ \\
Se atreven a cometer errores & 5 & $38 \%$ \\
Tienen un puesto alto & 2 & $15 \%$ \\
Otro & 2 & $15 \%$ \\
\hline Total & 13 & $100 \%$ \\
\hline
\end{tabular}

Tabla 5. Riesgo y Rentabilidad

\begin{tabular}{lll}
\hline Relación entre el riesgo y la rentabilidad esperada & & \\
\hline & n & $\%$ \\
A mayor riesgo en el proyecto, mayor rentabilidad & 7 & $54 \%$ \\
A mayor riesgo del proyecto, menor rentabilidad & 3 & $23 \%$ \\
Sin correlación & 3 & $23 \%$ \\
Total & 13 & $100 \%$ \\
\hline
\end{tabular}

La segunda versión de la relación de riesgo-rendimiento utiliza una visión retrospectiva del supuesto. En tal caso, los tomadores de decisiones que formaron parte del trabajo de campo trabajaban en ese momento en un proyecto y estaban experimentando el rendimiento del proyecto en curso. A la luz de la interpretación de un escenario contextual, tendrían que asumir una toma de decisiones arriesgada. Así, a los participantes se les preguntó las opciones respecto a la toma de decisiones arriesgadas en dos escenarios.

Para el escenario "el rendimiento de un proyecto en ejecución está por debajo de las expectativas", la mayoría de los participantes piensan que tienen que adoptar una postura más agresiva asumiendo una toma de decisiones con mayor riesgo (77\%). En este sentido, los supuestos señalados en la literatura relacionados con "cuanto menor es el rendimiento (en curso), se hace la decisión más riesgosa" coinciden con los resultados obtenidos de los participantes. Uno de ellos afirma lo siguiente: "Las decisiones conforme ya se están ejecutando los proyectos suelen ser más riesgosas, porque por lo general es más fácil corregir en la planeación, a en una etapa de fabricación".

De forma similar, para el escenario "el rendimiento de un proyecto en ejecución está por encima de las expectativas”, la mayoría de los participantes (69\%) tienden a tomar decisiones menos arriesgadas. Los resultados coinciden con trabajos previos (ver tabla 2) que indican que bajo este escenario, las conductas asumidas son más conservadoras.

En este sentido los resultados obtenidos de los gestores de proyectos mexicanos, se complementan con los resultados encontrados por Zwikael y Ahn (2011) en Nueva Zelanda, Israel y Japón. En donde se evidencia que los esfuerzos en la planificación de la gestión de riesgos son efectivos solo cuando el nivel de riesgo del proyecto es de medio a alto. En proyectos con bajos niveles de riesgo, la planificación del manejo de riesgos es ineficaz y puede ser innecesaria, lo que indica que los gerentes que supervisan los proyectos de alto riesgo invierten preferentemente más esfuerzos de planificación en un intento de enfrentar el riesgo.

\section{Gestión del riesgo}

En relación con el método utilizado para tomar decisiones riesgosas, lo encontrado indica que la mayoría de los participantes emplean un análisis simple (62\%), hubo quien selecciona el método en función de la decisión y quien emplea un método de análisis "pero si no obtengo un resultado definitivo e inefable sigo mi intuición”. Cabe señalar que también se considera la intuición para asumir las decisiones riesgosas (23\%) y solo un $15 \%$ utilizan técnicas avanzadas en donde se involucran análisis cuantitativos. Asimismo, en relación con la opinión sobre el control del riesgo, la mayoría indica que el riesgo se puede controlar: "siempre y cuando sea analizado [...] pero no eliminar al 100\%"; "si se toman las acciones pertinentes" y "siempre y cuando tenga datos" o información.

Estos resultados son similares a los obtenidos en Suecia por Riabacke (2006) y Hartono et al. (2014), sobre el uso limitado de técnicas cuantitativas. En contraste con las evidencias encontradas por Zwikael y Ahn (2011) en Nueva Zelanda, en donde la mayoría de los gerentes de proyectos utilizan herramientas de gestión de riesgos y específicamente en proyectos de ingeniería.

En estudios previos se han analizado las situaciones en las que se dispone de información (Hartono et al., 2014). En consecuencia, para una toma de decisión arriesgada mejor informada, los responsables de tomar decisiones racionales, comprenden la importancia de la adquisición de la información pertinente y oportuna a fin de minimizar los riesgos. A este respecto los participantes en primer lugar asignan la prioridad de "determinar varios aspectos pertinentes para resolver el problema” y "reunir más información", lo que implica que son conscientes de la importancia de la información.

Adicionalmente, los participantes también demuestran cierto grado de proceso deliberativo en la toma de decisiones según señalan la importancia de "determinar varios aspectos pertinentes para el problema”. Sin embargo, el hecho de que algunos de ellos se apoyen en sus intuiciones y pasen por alto la utilización de un análisis cuantitativo del riesgo, sugiere que aún no se ha aplicado plenamente la gestión de los riesgos en los proyectos analizados. 
Finalmente, a la luz de los hallazgos y únicamente considerándolos para la muestra analizada, se aceptan las hipótesis planteadas 2 y $3, y$, se rechazan las hipótesis 1 y 4 (ver tabla 6).

Tabla 6. Contrastación de Hipótesis

\begin{tabular}{|c|c|c|c|}
\hline & Hipótesis & Result & \\
\hline $\mathrm{H} 1$ & $\begin{array}{l}\text { La percepción del riesgo para más del } 50 \% \text { de los } \\
\text { profesionales que lideran proyectos comprende solo } \\
\text { una dimensión del riesgo, la negativa. }\end{array}$ & $38 \%$ & - \\
\hline $\mathrm{H} 2$ & $\begin{array}{l}\text { La actitud ante el riesgo para más del } 50 \% \text { de los } \\
\text { profesionales es moderada. }\end{array}$ & $62 \%$ & + \\
\hline H3 & $\begin{array}{l}\text { Los líderes de los proyectos perciben que hay una } \\
\text { relación entre el nivel de riesgo y el nivel de ren- } \\
\text { tabilidad. }\end{array}$ & $54 \%$ & + \\
\hline $\mathrm{H} 4$ & $\begin{array}{l}\text { Más del } 50 \% \text { de los líderes de proyectos utilizan téc- } \\
\text { nicas cuantitativas para el análisis de riesgos. }\end{array}$ & $15 \%$ & - \\
\hline
\end{tabular}

\section{Conclusiones}

Este trabajo analiza la interrelación conceptual entre el riesgo y la innovación, destacando que la asunción de riesgos en los proyectos de innovación es esencial para el crecimiento y creación de valor en las organizaciones. Así, el riesgo y por tanto la actitud de los líderes de las organizaciones ante éste, constituyen un factor crítico en el desarrollo de proyectos empresariales y especialmente en aquellos que involucran algún tipo de innovación.

La aplicación de un cuestionario entre gestores de proyectos revela que alrededor de una tercera parte de participantes, percibe el riesgo de manera negativa, porcentaje menor al que se observa en otros estudios de la literatura realizados en Estados Unidos, Israel e Indonesia. Es interesante destacar que incluso un grupo de ellos manifiesta no ser averso al riesgo. Lo anterior se justifica por el hecho de que los sujetos del estudio son líderes de proyectos de innovación, por lo que en general están más familiarizados con el riesgo.

Por otro lado, es relevante que una tercera parte de gestores de proyectos innovadores, asocia el concepto del riesgo a una perspectiva dual, lo que en la literatura se identifica como una perspectiva que propicia y da lugar al crecimiento en las empresas. Asimismo, respecto a la toma de decisiones, los participantes realizan comentarios vinculados a los pronunciamientos realizados por el PMI, lo que implica una vinculación estrecha entre las recomendaciones realizadas en la literatura con el ejercicio realizado en la práctica profesional.

No obstante, casi una tercera parte de los participantes no asocia el concepto del riesgo con lo señalado en el marco teórico del tema y un porcentaje similar afirma que no hay relación entre el riesgo y rentabilidad. Lo que delinea un grupo de gestores y líderes de proyectos en innovación que requieren enriquecer sus conocimientos de los principios teóricos y trabajos empíricos que sustentan la gestión del riesgo. Por lo que es relevante difundir la necesidad de educación continua en el ámbito profesional. Pues si bien, se reconoce que todo proyecto implica riesgos y ello se asocia a un impacto económico y a la generación de innovaciones, los profesionales que definen el riesgo con una perspectiva limitada pueden dar lugar a errores sistemáticos de juicio en los proyectos.

Asimismo, ante el hecho de que la mayoría de los participantes se apoyan en métodos simples o en la intuición para gestionar el riesgo, destacamos la necesidad de generar material académico recogido de la literatura previa, que incluya marcos de referencia y normativas de riesgo, modelos, métodos y herramientas, con base en el cual se ofrezca una visión más amplia para los futuros gestores de riesgos.

En cuanto a las limitaciones, destacamos que el muestreo por conveniencia utilizado, si bien es efectivo en términos prácticos, puede sesgar los resultados; por tanto, se recomienda aumentar el tamaño muestral y utilizar una muestra aleatoria, lo que posibilitaría la comparación entre los resultados obtenidos en estudios relacionados con diferencias entre países o tipos de proyectos. Estas diferencias no son el foco de este artículo; sin embargo, varios estudios identificados en la literatura previa, sugieren que las diferencias culturales entre los países a nivel mundial pueden ser útiles para evaluar el comportamiento agresivo frente al conservador, $y$, como un tópico vigente en la agenda de investigación, continua la evaluación de estos comportamientos en proyectos innovadores.

\section{Bibliografía}

Benhabib, J., Perla J., \& Tonetti, C. (2014). Catch-up and fall-back through innovation and imitation. Journal of Economic Growth, 19(1), 1-35. doi:10.1007/s10887-013-9095-z.

Bowers, J., \& Khorakian, A. (2014). Integrating risk management in the innovation project. European Journal of Innovation Management, 17(1), 25-40. doi:10.1108/EJIM-01-2013-0010.

Fernández-Mesa, A., Alegre-Vidal, J., \& Chiva-Gómez, R. (2012). Orientación emprendedora, capacidad de aprendizaje organizativo y desempeño innovador. Journal of Technology Management and Innovation, 7(2), 157-170. doi: http://dx.doi.org/10.4067/S071827242012000200013

García-Granero, A., Llopis, O., Fernández-Mesa, A., \& Alegre, J. (2015). Unraveling the link between managerial risk-taking and innovation: The mediating role of a risk-taking climate. Journal of Business Research, 68, 1094-1104. doi: 10.1016/j.jbusres.2014.10.012.

Hartono, B., Sulistyo, S., Praftiwi, P., \& Hasmoro, D. (2014). Project risk: Theoretical concepts and stakeholders' perspectives. International Journal of Project Management, 32(3), 400-411. doi: 10.1016/j. ijproman.2013.05.011. 
Hillson, D. (2002). Extending the risk process to manage opportunities. International Journal of Project Management, 20(3), 235-240. doi: 10.1016/S0263-7863(01)00074-6.

Holton, G. A. (2004). Defining risk. Financial Analysts Journal, 60(6), 19-25. doi:10.2469/faj.v60.n6.2669

International Standard Organisation, ISO (2009) 31000: 2009, Risk Management_Principles and Guidelines. Primera edición, Geneva.

Ling, Y., Simsek, Z., Lubatkin, M., \& Veiga, J. (2008). Transformational leadership's role in promoting corporate entrepreneurship: Examining the CEO-TMT interface. Academy of Management Journal, 21(3), 557-576. doi: 10.5465/amj.2008.32626023

Maccrinnon, K. R., \& Wehrung, D. (1990). Characteristics of risk taking executives. Management Science, 36, 422-435. doi:10.1287/ mnsc.36.4.422

March, J. G., \& Shapira, Z. (1987). Managerial perspectives on risk and risk taking. Management Science, 33, 1404-1418. doi:10.1287/ mnsc.33.11.1404

Organisation for Economic Co-operation and Development [OECD] \& Eurostat. (2005). Oslo Manual. Paris: OECD. Doi: 10.1787/19900414

Peterson, R. S., Smith, D.B., Martorana, P. V., \& Owens, P. D. (2003).

The impact of chief executive officer personality on top management team dynamics: One mechanism by which leadership affects organizational performance. Journal of Applied Psychology, 88, 795-808. doi: 10.1037/0021-9010.88.5.795.

Project Management Institute (2013). Guía de los Fundamentos Para la Dirección de Proyectos (Guía del PMBOK ${ }^{\circledR}$ )-Quinta Edición (SPANISH). Project Management Institute.

Porter, M. E. (1990). The Comparative Advantage of Nations. New York: Free Press. doi: 10.1007/978-1-349-11336-1

PwC, \& IFAC (1999). Enhancing Shareholder Wealth by Better Managing Business Risk. New York: PricewhaterhouseCoopers and International Federation of Accountants.
Rauch, A., Wiklund, J., Lumpkin, G. T., \& Frese, M. (2009). Entrepreneurial orientation and business performance: An assessment of past research and suggestions for the future. Entrepreneurship: Theory and Practice, 33(3), 761-787. doi: 10.1111/j.15406520.2009.00308.x.

Rabechini Junior, R., \& Monteiro de Carvalho, M. (2013). Understanding the impact of project risk management on project performance: An empirical study. Journal of Technology Management \& Innovation, 8, 64-78. doi: 10.4067/s0718-27242013000300006

Riabacke, A. (2006). Managerial Decision Making Under Risk and Uncertainty. IAENG International Journal of Computer Science, 32(4). Roussel, P., Saad, K., \& Erickson, T. (1991). Third generation R\&D: Managing the link to corporate strategy. Harvard: Harvard Business School Press.

Taplin, R., \& Schymyck, N. (2005). An interdisciplinary and crosscultural approach. In Taplin, R. (Ed.), Risk Management and Innovation in Japan, Britain and the United States. London: Routledge, 1-20. doi:10.4324/9780203027783

Thevendran, V., \& Mawdesley, M. J. (2004). Perception of human risk factors in construction projects: an exploratory study. International Journal of Project Management, 22(2), 131-137. doi: 10.1016/s02637863(03)00063-2

Vargas-Hernández, J. G. (2011). Modelling risk and innovation management. Advances in Competitiveness Research, 19(3/4), 45-57.

Verbano, C., \& Venturini, K. (2013). Managing risks in SMEs: A literature review and research agenda. Journal of Technology Management \& Innovation, 8(3), 186-197. doi: 10.4067/s071827242013000400017

Wu, H. L. (2008). When does internal governance make firms innovative? Journal of Business Research, 61(2), 141-153. doi:10.1016/j. jbusres.2007.06.010.

Zwikael, O., \& Ahn, M. (2011). The effectiveness of risk management: an analysis of project risk planning across industries and countries. Risk analysis, 31(1), 25-37. doi: 10.1111/j.1539-6924.2010.01470.x. 
